\title{
Antigen-Presentation Machinery Expression Profile
}

National Cancer Institute

\section{Source}

National Cancer Institute. Antigen-Presentation Machinery Expression Profile. NCI

Thesaurus. Code C147085.

The identification and quantitation of all of the antigen-presentation machinery

molecules expressed in a biological sample. 